\title{
High Payload Qr-Based Data Hiding Using Secured Compressed Watermark in Polar Domain
}

\author{
Indrarini Dyah IRAWATI ${ }^{\text {a }, ~} 1$, Gelar BUDIMAN ${ }^{\text {b }}$, Kholidiyah MASYKUROH ${ }^{\mathrm{c}}$, Zein \\ Hanni PRADANA ${ }^{\mathrm{c}}$ and Arfianto FAHMI ${ }^{\mathrm{b}}$ \\ a School of Applied Science, Telkom University, Indonesia \\ ${ }^{b}$ School of Electrical Engineering, Telkom University, Indonesia \\ ${ }^{c}$ Fakultas Teknik Telekomunikasi dan Elektro, Institut Teknologi Telkom Purwokerto, \\ Indonesia
}

\begin{abstract}
Audio Watermarking is a method to insert a copyright marker on audio. This method inserts a watermark in the information form and in a way that does not damage the audio. This technique is one of the ways to solve the problem of copyright infringement. The embedded watermark has to meet the condition of not damaging the audio and must have robustness, imperceptibility, and good capacity. The data hiding technique use the combined method of Lifting Wavelet Transform (LWT), Fast Fourier Transform (FFT), QR Decomposition and Reconstruction, and Cartesian-Polar Transformation (CPT) based on Quantization Index Modulation (QIM) with the secured and compressed watermark using Compressive Sampling (CS) technique. The proposed scheme is blind Audio Watermarking as it no needs for original audio in the detection process. The combination of methods overcomes multiple attacks with guaranteed quality watermarking and high capacity. Compared to the existing technique, the data hiding technique can withstand LPF attacks, Resampling, Linear speed change (LSC), and MP3 compression. This proposed technique is also secured due to the coded watermark by a particular random key using CS. Combining CS and Audio Watermarking techniques can perform well in capacity, imperceptibility, security, and attack resistance.
\end{abstract}

Keywords. Audio Watermarking, CPT, CS, FFT, LWT, QR Decomposition, QIM

\section{Introduction}

Digital watermarking is inserting digital watermarks on information such as audio signals, images, or documents used as copyright markers [1]. The insertion of a watermark on the information must not destroy the original data. Audio Watermarking is an application that is compatible with the Human Auditory System (HAS). In research [2], Audio Watermarking schemes can maintain copyright security and are robust against

1 Corresponding author, School of Applied Science, Telkom University, Indonesia; E-mail: indrarini@telkomuniversity.ac.id. 
various standard signal processing attacks such as noise addition, filtering, quantization, echo, invert, and compression.

Audio Watermarking research has been developed by several previous researchers [2-17]. Singha and Ullah propose a combination of multi-level Discrete Wavelet Transform (DWT) and Singular Value Decomposition (SVD) on multiple images of different sizes as a decentralized watermark [3]. Chen and Wornell used the Quantization Index Modulation (QIM) technique which has good performance on the parameters of embedding robustness. The degradation of the host signal can solve by removing interference [4].

Research [5-9] exploited an Audio Watermark using the Lifting Wavelet Transform (LWT) method. In [5], Hu and Lee explored LWT to level 3 decomposition for blind audiwatermarks capable of overcoming cropping and alternation attacks, while effectively detecting tamper and self-recovery. Another study combines audio watermarking techniques, namely Cordic QR decomposition and multi-resolution decomposition to produce a good balance of perceptual transparency, robustness, and payload. [6]. A study by Lei combines the LWT-DCT-SVD method. The method has resistant to hybrid and desynchronization attacks with good imperceptibility [7]. Budiman [8] proposed Audio Watermarking using QR and CPT in the SWT-DST domain with high imperceptibility and good robustness to MP3 compression and resampling. However, its payload is still low or less than $100 \mathrm{bps}$. LWT with the Spread Spectrum method and has the best optimization results can only be done on optimized audio, has poor robustness against some attacks. In the study [9], the authors combined the LWT-QR factorization based on QIM. The correlation coefficient result can increase the security of the watermarking algorithm.

The DWT technique is also applied to Audio Watermarking by the authors $[3,10$ 11]. For example, paper [10] proposes a hybrid watermarking algorithm for voice signal cryptography to protect the copyright of audio files. The algorithm is formed by combining three-domain transformations, DCT, DWT, and SVD, increasing the strength of resistance to audio processing attacks. Another study combined second-level 2D-Haar DWT and Schur decomposition. This hybrid scheme has succeeded in increasing imperceptibility, robustness, and data payload size [11].

In [12], the authors proposed a combined transform LWT-DCT, then decomposed by QR, then embedded by QIM. The result is good imperceptibility and high capacity, but the robustness is still moderately low against LPF and resampling attacks. Looking for performance differences between FFT and DCT using the Fibonacci sequence of numbers to generate high capacity, deliver robustness against common signal processing attacks, and cause very low perceptual distortion. Irawati et al. combined audio watermarking techniques based on QIM with LWT, DCT, QR Decomposition. The combined performance of the methods results in a powerful and invisible audio watermark with a very high watermark payload [13]. In [14], the authors presented an audio watermarking with a CS scheme. The audio host is embedded by the watermark and compressed by the CS technique using a multi-bit spread spectrum (SS) framework. Research by Kaur et al. inserted watermarking bits using the embedding function on each frame in the $\boldsymbol{R}$ matrix [15]. Patel and Amin performed CS for optimizing signal reconstruction in Audio Watermarking [16]. CS samples the image before embedding the image into the audio host by QIM. The audio host is represented as a one-dimensional matrix. Then LWT decomposes a one-dimensional matrix into some sub-bands. The lowfrequency sub-band will be further processed. The DCT technique converts the signal 
into essential frequency components. The QR factorization decomposes each DCT frame into an orthogonal matrix $(\mathrm{Q})$ and an upper triangle $(\mathrm{R})$.

In this study, we proposed a secured-compressed watermark system based on QR. The proposed method is a combination of several techniques. First, a host is decomposed with LWT, and then the signal is converted into the frequency domain with FFT. Next, the signal converted into the frequency domain is converted into the $\boldsymbol{M} \times \boldsymbol{M}$ matrix to retrieve the $\boldsymbol{R}$ matrix with a QR decomposition. After the signal becomes a matrix, each element in the matrix will be changed into a polar form using the Cartesian-Polar Transform (CPT). The watermark image is altered from a two-dimensional state to a onedimensional structure, and after that, the CS process 1 is the same as in [17] to obtain watermark compression. After the watermark is pre-processing and CS, each watermark bit is inserted into each matrix element using QIM. After all the watermark bits are inserted, the inverse process in ICPT is carried out to return the bits in the R matrix element into cartesian form and proceed by reconstructing the $\mathrm{R}$ and $\mathrm{Q}$ matrix with $\mathrm{QR}$ reconstruction. After that, the $\boldsymbol{M} \times \boldsymbol{M}$ matrix is converted back into a 1-dimensional signal again by IFFT into a back-time domain. Finally, ILWT changes the reconstructing of the signal into complete audio again. LWT decomposes the audio in the extraction process, then converted into the frequency domain by FFT. The 1-dimensional signal is converted into an $\boldsymbol{M} \times \boldsymbol{M}$ matrix to get the $\boldsymbol{R}$ matrix from QR decomposition. Then CPT is performed to convert each element into a polar shape. Then after the CPT is carried out on each component in the $\boldsymbol{R}$ matrix, the extraction process is carried out by QIM. QIM extracted CS reconstructed bits, and post-processing was carried out to obtain the perfect watermark shape. Those methods are expected to result in high robustness to noise addition, cropping, resampling, signal addition, signal reduction, and MP3 compression.

The next section of the paper is described as follows: section 2 illustrates the basic theory of Audio Watermarking, transformation technique, and CS. Section 3 explained the embedding and extraction process of the Audio Watermarking system. While section 4 presented the analysis result of the proposed Audio Watermarking system with scenarios before and after attacks. The last section is the conclusion and future works.

\section{Basic Theory}

This paper used LWT to select bits as the selection point. FFT method to change the domain host to the frequency domain, QR Decomposition method to build the matrix, Cartesian Polar Transform method to change the polar form to cartesian form. CS method to compress the watermark before embedded, and Quantization Index Modulation to embed the watermark.

\subsection{Lifting Wavelet Transform}

LWT is faster than traditional Wavelets and can generate unsuitable resolution analyses on a uniform grid $[18,19]$. The Lifting Wavelet scheme process is divided into three steps: split, predict, and update.

- $\quad$ Split 
Split is a step to divide the audio host signal that has been segmented into two-part, where the initial sequence is the odd part, and the final half order is the even part, as the following equation explains both sections.

$$
\begin{gathered}
x[n]=x_{e}[n]+x_{o}[n] \\
x_{e}[n]=x[2 n] \\
x_{o}[n]=x[2 n+1]
\end{gathered}
$$

where $x[n]$ is the segmented audio signal, notation $x_{e}[n]$ does the even part take the segmented signal, $x_{o}[n]$ is the segmented signal taken by the odd part, and $n$ is the time domain.

- Prediction

After the signal is divided into two, then the prediction is made by using even sequence correlation and odd sequence shown by Wavelet Coefficient $(d[n])$. Wavelet Coefficient is obtained by calculating the original value (odd) minus the prediction value (taken from the even part). The following equation explains the Prediction process.

$$
d[n]=x_{o}[n]-P\left(x_{e}[n]\right.
$$

where $d[n]$ is the coefficient of Wavelet and $P\left(x_{e}[n]\right)$ is the prediction operator of $x_{e}[n]$.

- Update

All subsets of split results have values that may differ from the original data. However, the general characteristics of the data must be kept consistent through the update process. The following equation describes the update process.

$$
c[n]=x_{e}[n]+U(d[n])
$$

where $c[n]$ is the lifted loop, and $U(d[n])$ is the prediction operator.

\subsection{Fast Fourier Transform}

The FFT algorithm is based on the principal principle of the decomposition of the discrete Fourier transform (DFT) calculation of a sequence along $\mathrm{N}$ into the DFT.

The FFT is defined by the formula [20]:

$$
H(k)=\sum_{n=0}^{\left(\frac{N}{2}\right)-1} h(2 n) W_{N / 2}^{n k}+\sum_{n=0}^{\left(\frac{N}{2}\right)-1} h(2 n+1)
$$

where $H(k)$ is the domain transformation value, the digital media value is notated by $h(n), k$ is the frequency domain, $n$ is the time domain, and $N$ is the amount of data converted into the frequency domain.

To perform inversion Fast Fourier Transform (IFFT) then it can do with Eq. (7):

$$
h(n)=\frac{1}{N} \sum_{n=0}^{\left(\frac{N}{2}\right)-1}\left[R e * \cos \left(\frac{2 \pi k n}{N}\right)+\operatorname{Im} * \sin \left(\frac{2 \pi k n}{N}\right)\right]
$$

where $R e$ being an actual number, $I m$ is an imaginary number, and the serial value of the watermark complex is notated by $h(n)$. 


\subsection{QR Decomposition}

The QR Decomposition of the quadratic matrix $\boldsymbol{A}$ is decomposition $\boldsymbol{A}$, which is defined by Eq. (8) [21,22]:

$$
\boldsymbol{A}=\boldsymbol{Q} \cdot \boldsymbol{R}
$$

where $\boldsymbol{Q}$ is an orthogonal matrix with $\boldsymbol{Q}^{\boldsymbol{T}} . \boldsymbol{Q}=1$ and $\boldsymbol{R}$ are an upper triangular matrix.

If $\boldsymbol{A}$ is a nonsingular matrix, then factorization is unique. There are several methods for calculating QR decomposition. One such method is the Gram-Schmidt process. Based on the Gram-Schmidt procedure, the matrix column $\boldsymbol{A}$ is considered as the processed vector as shown in (9).

$$
A=\left[a_{1}\left|a_{2}\right| \ldots \mid a_{n}\right]
$$

Based on the Gram-Schmidt process then the result of QR factorization is as follows.

$$
\begin{aligned}
A & =\left[a_{1}\left|a_{2}\right| \ldots \mid a_{n}\right] \\
& =\left[e_{1}\left|e_{2}\right| \ldots \mid e_{n}\right]\left[\begin{array}{cccc}
a_{1} \cdot e_{1} & a_{2} \cdot a_{1} & \ldots & a_{n} \cdot e_{1} \\
0 & a_{2} \cdot e_{2} & \ldots & a_{n} \cdot e_{2} \\
\vdots & \vdots & \ddots & \vdots \\
0 & 0 & \ldots & a_{n} \cdot e_{n}
\end{array}\right]=Q \cdot R
\end{aligned}
$$

where $a_{1} \ldots a_{n}$ is a column of matrices $\boldsymbol{A}$ and an orthonormal set in column matrices indicated in the notation $e_{1} \ldots e_{n}$.

\subsection{Cartesian-Polar Transform}

In the polar coordinate system $(r, \theta), r$ shows the distance from the origin, and $\theta$ shows the angle between the reference line and the line through the origin and endpoints. The transformation to polar coordinate is given by the Eq. (11) [8]:

$$
r=\sqrt{x^{2}+y^{2}}, \quad \theta=\tan ^{-1}\left(\frac{y}{x}\right)
$$

where $(x, y)$ is a point on the polar coordinate system. Transformations from polar coordinates to cartesian coordinates are given in Eq. (12)

$$
x=r \cos \theta, \quad y=r \sin \theta
$$

\subsection{Cartesian-Polar Transform}

Quantization Index Modulation (QIM) is a technique of inserting a Watermark depending on a signal by quantizing a host signal with a selected quantization based on the bit value of the message encoded.

In QIM, there is parameter $\Delta$ used as an insertion parameter. This parameter is based on the quantity of $n$ bit or bits quantization in the QIM method by calculating the distance between quantization. The following are the equations used for the insertion process and the extraction process on the watermark.

- QIM embedding process: 
If $w=1$ then,

$$
F^{\prime}(0)=A_{k} \text { and } \arg \min \left|F(0)-A_{k}\right|
$$

If $w=0$ then,

$$
F^{\prime}(0)=A_{k} \text { and } \arg \min \left|F(0)-A_{k}\right|
$$

where,

$$
A_{k}=\left(2 k+\frac{1}{2}\right) \Delta, B_{k}=\left(2 k-\frac{1}{2}\right) \Delta, k=0, \pm 1, \pm 2, \ldots \ldots
$$

- QIM extraction process:

$$
\begin{gathered}
\widetilde{w}(k)=\bmod \left(\operatorname{ceil}\left(\frac{F(0)}{\Delta}\right), 2\right) \\
\Delta=\frac{1}{2^{(n b i t-1)}}
\end{gathered}
$$

where $F(0)$ is the audio host sample before it is quantized, $F^{\prime}(0)$ is the audio host sample after embedded. $w$ is watermark bit before embedded, watermark bit after embedded or before extraction shown in the variable $\widetilde{w}$. Variable $\Delta$ is delta or the distance between the quantization values. Variable $k$ is the quantization index, and $\mathrm{n}$ bit is bit quantization on the QIM method.

\subsection{Compressive Sensing}

CS is a new signal processing paradigm. CS is also a technique that can reconstruct data from incomplete measurements. CS utilizes a unique structure in the data, namely data sparsity. The sparse data is represented with a few non-zero values [17].

A signal expressed in $R^{N}$ is represented in a vector base $N \times 1\left\{\psi_{i}\right\}_{i=1}^{N}$ An orthogonal form. Using the $N \times N$ base matrix $\boldsymbol{\psi}$ with $\left\{\psi_{i}\right\}_{i=1}^{N}$ like the column, the actual value of the dimension signal $f$ can write with the following equation.

$$
f=\boldsymbol{\psi} x
$$

An $x$ is said to be "K-sparse" only if $K$ comes from the coefficient $x_{i}$, which is a non-zero coefficient when $(N-K)$ is zero, then $K \ll N$. If the linear measurement process is general, then $M \times N$ on the matrix $\boldsymbol{\phi}(M \ll N)$ will satisfy.

$$
y=\boldsymbol{\Phi} f
$$

where $y$ is the vector $M \times 1$, the measurement vector, and $\boldsymbol{\Phi}$ is the matrix $M \times N$. Based on the first Eq 1, it can be formulated into

$$
y=\boldsymbol{\Phi} f=\boldsymbol{\Phi} \boldsymbol{\psi} x=\boldsymbol{\Theta} x
$$

where $\boldsymbol{\Theta}=\boldsymbol{\Phi} \boldsymbol{\psi}$ is an $M \times N$ matrix known as the CS matrix. As for CS reconstruction with the minimum of $\ell_{1}$ can be shown in the following formulas [17].

$$
\min _{x}\|x\|_{l 1} \text { subject to } y=\boldsymbol{\Phi} x
$$


where $l 1$-norm of the signal $x$ is the absolute value of the sum of the signal samples and is expressed in the following equation:

$$
\|x\|_{l 1}=\sum_{i=1}^{N}\left|x_{i}\right|
$$

\section{Watermarking Model}

This section explains the modeling system used, which begins with the embedding process. Various attacks will attack it after it does the extraction process, and then it calculates the quality output of the Audio Watermarking.

\subsection{Embedding Process}

The embedding process has three steps. There is a bit selection process as insertion place, host domain transformation, and bit watermark insertion process. Figure 1 shows the embedding process flow chart.

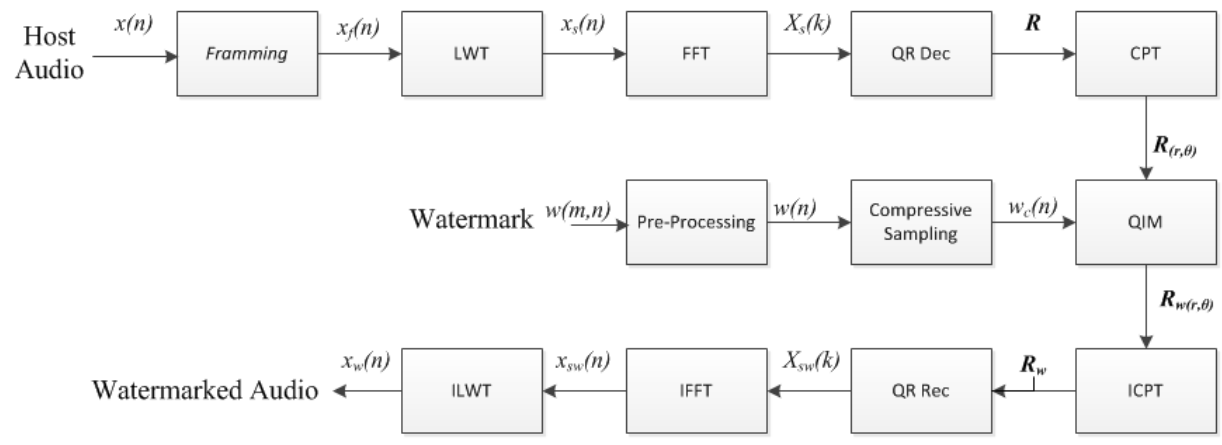

Figure 1. Embedding Process Flow Chart.

\subsection{Extraction Process}

After we attack watermarked audio, the watermarked audio data will extract. The step of extracting process is indicated in Figure 2:

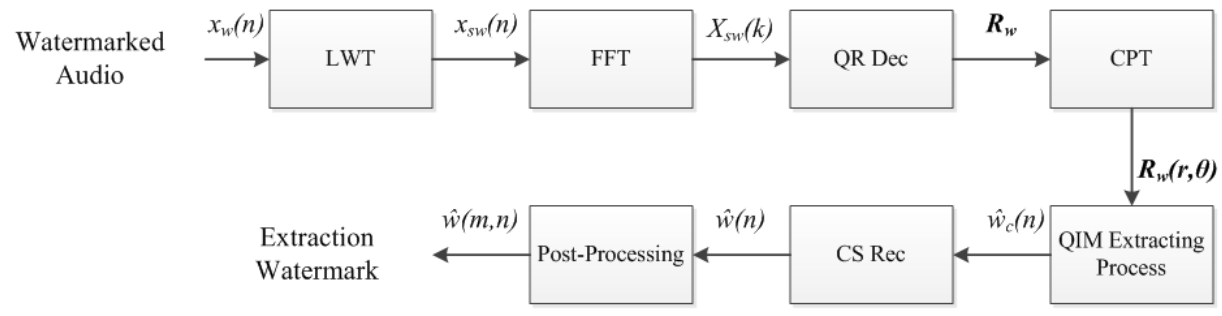

Figure 2. Extraction Process Flow Chart. 


\section{Simulation Result}

In the simulation, we have input parameters such as nbit as bit number of QIM, Nframe as sample number per segment, and $N$ as decomposition level. These parameters affect the output or performance parameters such as ODG and SNR as watermarked audio quality, $C$ as watermark payload, and BER as watermark robustness. We apply the simulation with the change of parameters to understand the relation between the input parameters and the performance parameters. We use an image for the watermark, with a resolution of 10x15 pixels. We also use four audio files such as piano.wav, voice.wav, guitar.wav, and bass.wav as host files. The host file sampling frequency is $44.1 \mathrm{kHz}$ with 16-bit audio quantization. The simulation results are shown in the graph in the Figure 3, Figure 4, and Figure 5.

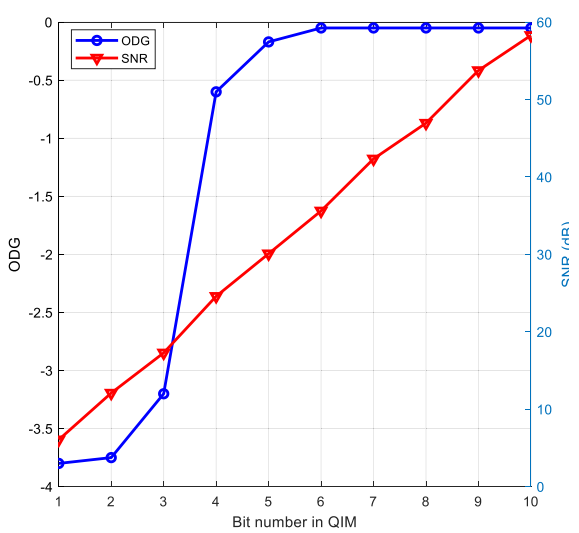

Figure 3. The $n$ bit effect ODG and SNR.

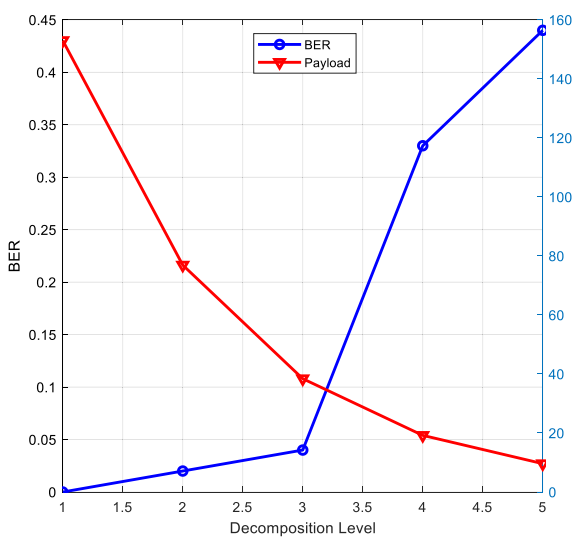

Figure 4. The $N$ effect to BER and payload.

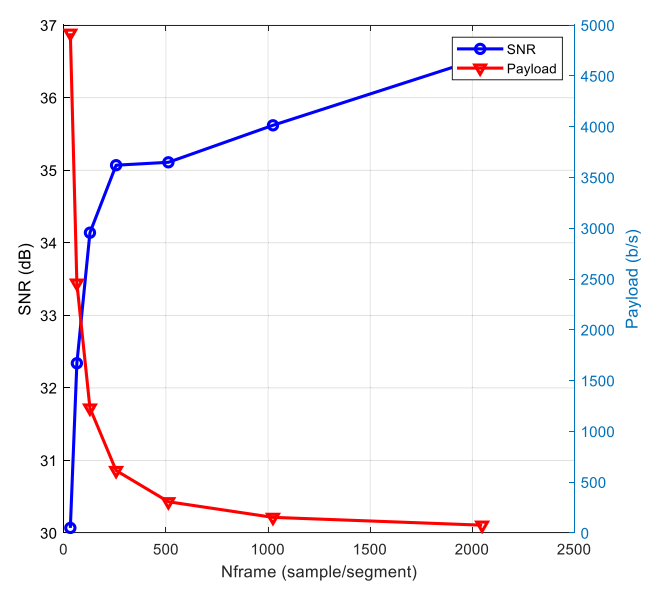

Figure 5. The $N$ frame effect to SNR and payload.

The first simulation with voice.wav as a host file changes nbit from 1 to 10 bits while $N=1$ and $N$ frame $=1024$ sample/segment. These parameters obtain the watermark payload $C=153$ bps with the minimum, and maximum BER is 0 and 0.18 , respectively. The audio quality represented by SNR and ODG is displayed in Figure 3. The rise of 
nbit increases the audio quality in ODG and SNR. This improvement is because of the reduction of modified audio amplitude by QIM when nbit increases. However, the PEAQ procedure calculates ODG with the perceptual process. Thus, the rise of ODG is not linear compared to the increase of SNR. For example, analyzing Figure 1, we can select minimum nbit $=4$ due to the $\mathrm{SNR}>20 \mathrm{~dB}$ and $\mathrm{ODG}>-1$.

The second simulation with voice.wav as a host file then changes decomposition level or $N$ from 1 to 5 while $N$ frame $=1024$ sample/segment and $n b i t=6$. The simulation result with these parameters shows in Figure 5 and obtains SNR 35.62 to $42.82 \mathrm{~dB}$ and ODG -0.05 to -0.80 . Figure 4 shows that the trend in $\mathrm{N}$ is aligned with the BER value and opposite to the watermark payload. The larger N, the BER increases but the payload of the watermark decreases. This fluctuation illustrates the trade-off between the durability and payload of the watermark when the decomposition rate changes. We can select the maximum decomposition level $N=3$ due to $\mathrm{BER}<10 \%$.

The third simulation with voice.wav as a host file changes Nframe from 32 to 2048 while $N=1$ and $n b i t=6$. BER $=0$ and ODG 0.08 to -2.59 are the parameters that can obtain. Figure 5 displays the effect of Nframe on the watermarked audio quality and the watermark payload. By increasing Nframe, the payload decreases but on the other hand, the audio quality or SNR increases. This fluctuation displays the trade-off between the watermarked audio quality and the watermark payload when Nframe changes. From Figure 5, we can choose all values of Nframe 32 to 2048 since the payload is still more than 20 bps and the audio quality is still more than $20 \mathrm{~dB}$. Thus, we can select a certain Nframe that is robust to a specific attack.

Table 1. Watermark robustness test with BER parameter.

\begin{tabular}{|c|c|c|c|c|c|}
\hline \multirow[t]{2}{*}{ Attacks } & \multirow[t]{2}{*}{ Parameter } & \multicolumn{4}{|c|}{ BER } \\
\hline & & Voice & Piano & Guitar & Bass \\
\hline \multirow[t]{3}{*}{ LPF } & $3 \mathrm{k}$ & 0 & 0.011 & 0.019 & 0 \\
\hline & $6 \mathrm{k}$ & 0 & 0.011 & 0 & 0 \\
\hline & $9 \mathrm{k}$ & 0 & 0.011 & 0 & 0 \\
\hline \multirow[t]{3}{*}{ BPF } & $100-6 \mathrm{k}$ & 0.312 & 0.195 & 0.423 & 0.45 \\
\hline & $50-6 \mathrm{k}$ & 0.093 & 0.05 & 0.335 & 0.253 \\
\hline & $25-6 \mathrm{k}$ & 0.023 & 0.046 & 0.328 & 0.089 \\
\hline \multirow[t]{3}{*}{ Noise } & $0 \mathrm{~dB}$ & 0.468 & 0.511 & 0.464 & 0.484 \\
\hline & $10 \mathrm{~dB}$ & 0.464 & 0.460 & 0.472 & 0.437 \\
\hline & $20 \mathrm{~dB}$ & 0.222 & 0.25 & 0.238 & 0.394 \\
\hline \multirow[t]{3}{*}{ Resampling } & $22.05 \mathrm{k}$ & 0 & 0.011 & 0 & 0 \\
\hline & $11.025 \mathrm{k}$ & 0 & 0.011 & 0 & 0 \\
\hline & $16 \mathrm{k}$ & 0 & 0.011 & 0 & 0 \\
\hline \multirow[t]{3}{*}{ LSC } & $1 \%$ & 0 & 0.023 & 0.019 & 0 \\
\hline & $5 \%$ & 0.031 & 0 & 0.011 & 0 \\
\hline & $10 \%$ & 0.031 & 0 & 0.011 & 0 \\
\hline Equalizer & & 0.148 & 0.210 & 0.312 & 0.328 \\
\hline \multirow[t]{4}{*}{ MP3 Compression } & $32 \mathrm{k}$ & 0.093 & 0.109 & 0.148 & 0.128 \\
\hline & $64 \mathrm{k}$ & 0.058 & 0.046 & 0.074 & 0.027 \\
\hline & $128 \mathrm{k}$ & 0 & 0 & 0.019 & 0.027 \\
\hline & $192 k$ & 0 & 0 & 0.019 & 0 \\
\hline Average & & & 0.204 & & \\
\hline
\end{tabular}


We apply the fourth simulation by parameters $N=1, N$ frame $=512$ sample/segment, and $n b i t=4$. This parameter yields the average ODG -1.79 and SNR $23.27 \mathrm{~dB}$ with payload $307.62 \mathrm{bps}$. Finally, we apply our proposed method of several attacks, as displayed in Table 1. The watermark robustness testing shows that the method we developed is resistant to various attacks, including LPF, resampling, LSC, and MP3 compression using $128 \mathrm{kbps}$ bit rate.

BER is a watermark resistance test parameter. This value describes the quality of the extracted watermark image from the attacked Audio Watermark. The higher the BER, the worse the quality of the extracted watermark image, and vice versa. There is a maximum BER threshold on the damaged watermark image, but it can still be accepted and understood by humans visually. The resolution of the watermark image that is Table 2 shows the watermark image from the original image in Figure 3. with a resolution of $10 \times 15$ and BER $=0$. The extracted watermark can be read even though the watermark image is damaged in the range of BER $=0.011$ to 0.074 . While the BER of the watermark image is $12.8 \%$ and above, the extracted watermark is unreadable. This condition shows the watermark robustness is acceptable when the BER is up to $7.4 \%$.

Table 2. Visualization of Watermark Extraction.

\begin{tabular}{|c|c|c|c|}
\hline BER & Watermark Extraction & BER & Watermark Extraction \\
\hline 0 & & 0.058 & \\
\hline 0.011 & & 0.074 & \\
\hline 0.027 & & 0.128 & \\
\hline 0.046 & & 0.222 & \\
\hline
\end{tabular}

Table 3 describes performance comparison between our proposed method and the previous method using the QR decomposition as one of the processes before embedding. Our proposed method has relatively better in terms of robustness with high capacity, despite lower capacity than [13]. Nevertheless, the robustness in [13] is too low compared to our proposed method's robustness. NA from the table means not available or not reported. The imperceptibility of our method is the lowest, but it is still accepted due to more than the standard minimum of audio quality or $20 \mathrm{~dB}$ [23].

Table 3. Performance Comparison.

\begin{tabular}{|c|c|c|c|c|c|c|}
\hline \multirow[t]{2}{*}{ Ref. } & \multicolumn{4}{|c|}{ Robustness/BER (\%) } & \multirow{2}{*}{$\begin{array}{l}\text { Impercepti } \\
\text { bility/SNR } \\
\text { (dB) }\end{array}$} & \multirow[t]{2}{*}{ Capacity (bps) } \\
\hline & $\begin{array}{c}\text { MP3 } \\
64 k \\
\text { bps }\end{array}$ & $\begin{array}{l}\text { MP3 128k } \\
\text { bps }\end{array}$ & LPF & $\begin{array}{c}\text { Resampling } \\
22.05 \mathrm{kHz}\end{array}$ & & \\
\hline [8] & 9 & 3 & NA & $0-1$ & 32.72 & 21.43 \\
\hline [13] & $0-14$ & $0-5$ & $0-14$ & $0-14$ & 31.40 & 615.23 \\
\hline Proposed & $2.7-7.4$ & $0-2.7$ & $0-1.1$ & $0-1.1$ & 23.25 & 307.62 \\
\hline
\end{tabular}




\section{Conclusion}

We propose the Audio Watermarking technique with the combined Lifting Wavelet Transform, Fast Fourier Transform, QR Decomposition and Reconstruction, and Cartesian-Polar Transformation based on Quantization Index Modulation. The watermark is sampled using CS to reduce the embedded watermark size. The selected parameters from the simulation yield the average value of ODG is -1.79 , the average value of SNR is 23.35, and the watermark payload $307.62 \mathrm{bps}$. Using the selected parameters, the method we propose has a good performance as robust to various attacks, such as LPF, resampling, LSC, and MP3 compression with $128 \mathrm{kbps}$.

There are still opportunities to re-examine the attacks that have not been successfully anticipated using the proposed method. The more technology develops, the more attacks will appear, so it is necessary to explore the features to resist them. In addition, it is required to develop research on the effect of using CS on Audio Watermarks.

\section{Acknowledgment}

This research is supported by Telkom University, Telkom Institute of Technology Purwokerto, and Telkom Foundation.

\section{References}

[1] Abdulla NTB and Navas KA. High security watermarking techniques for digital rights management: a review. In: International Conference on Communication and Signal Processing (ICCSP); 2020 July 2830; Chennai, India: IEEE; c2020. p. 162-166.

[2] Nair U and Birajdar GK. Compressed domain secure, robust and high-capacity audio watermarking. Iran Journal of Computer Science. 2020; 3:217-232.

[3] Singha A and Ullah MA. Development of an audio watermarking with decentralization of the watermarks. Journal of King Saud University -Computer and Information Sciences. 2020 September; 1-7.

[4] Chen B and Wornell GW. Preprocessed and postprocessed quantization index modulation methods for digital watermarking. In: Proceeding Security and Watermarking of Multimedia Contents II; 2000 May 9; San Jose, CA, United States: 3971.

[5] Hu $\mathrm{H}$ and Lee T. Hybrid blind audio watermarking for proprietary protection, tamper proofing, and selfrecovery. IEEE Access. 2019 December; 7:180395-180408.

[6] Gupta A, Kaur A, Dutta MK and Schimmel J. Perceptually transparent \& robust audio watermarking algorithm using multi resolution decomposition \& cordic qr decomposition. In: International Conference on Telecommunications and Signal Processing (TSP); 2019 July 1-3; Budapest, Hungary: IEEE; c2019. p. 313-317.

[7] Lei B, Soon IY and Tan E. Robust SVD-based audio watermarking scheme with differential evolution optimization. IEEE Transactions on Audio, Speech, and Language Processing. 2013; 21(11):2368-2378.

[8] Budiman G, Suksmono AB, Danudirdjo D and Pawellang S. QIM-based audio watermarking with combined techniques of SWT-DST-QR-CPT using SS-based synchronization. In: International Conference on Information and Communication Technology (ICoICT); 2018 May 3-5; Bandung, Indonesia: IEEE; c2018. p. 286-292.

[9] Li J and Wu T. Robust audio watermarking scheme via QIM of correlation coefficients using LWT and QR decomposition. In: International Conference on Informative and Cybernetics for Computational Social Systems (ICCSS); 2015 August 13-15; Chengdu, China: IEEE; c2015. p. 1-6.

[10] Rezaei A, Khalili M. A robust blind audio watermarking scheme based on DCT-DWT-SVD. In Montaser Kouhsari, S, editor. Fundamental research in electrical engineering. Singapore: Springer. 2019. p. 101113.

[11] Karajeh H, Khatib T, Rajab L and Maqableh M. A robust digital audio watermarking scheme based on DWT and Schur decomposition. Multimedia Tools Application. 2019; 78:18395-18418. 
[12] Budiman G, Suksmono AB and Danudirdjo D. CPT-based data hiding in selected subband using combined transform and decomposition method. In: International Conference on Control, Electronics, Renewable Energy and Communications (ICCEREC). 2018 December 5-7; Bandung, Indonesia: IEEE; c2019. p. 86-92.

[13] Irawati ID, Budiman G and Ramdhani F. QR-based watermarking in audio subband using DCT. In: International Conference on Control, Electronics, Renewable Energy and Communications (ICCEREC); 2018 December 5-7; Bandung, Indonesia: IEEE; c2019.p. 136-141.

[14] Budiman G, Suksmono AB and Danudirdjo D. Compressive sampling with multiple bit spread spectrumbased data hiding. Applied Sciences. 2020 June;10(12): 4338-4359.

[15] Kaur A, Dutta MK, Soni KM and Taneja N. A high payload audio watermarking algorithm robust against Mp3 compression. In: 7th International Conference on Contemporary Computing (IC3); 2014 August 79; Noida, India: IEEE; c2014. p. 531-535.

[16] Patel R and Amin JD. Alpha channel base data hiding using compressive sensing. International Journal of Advance Research and Innovative Ideas in Education. 2016; 3:544-549.

[17] Huang H, Chang F, Lu Y and Pang Y. Multiple watermarking for compressed sensing with robust transmission applications. In: Global Conference on Consumer Electronics (GCCE). 2018 October 9-12; Nara, Japan: IEEE; c2018. p. 371-372.

[18] Tomar V, Kumar A and Choudhary A. Conception \& implementation of a novel digital image watermarking algorithm using cascading of DCT and LWT. In: International Conference on Reliability, Optimization and Information Technology (ICROIT). 2014 February 6-8; Faridabad, India: IEEE; c2014. p. 501-505.

[19] Ke WY and Jian W. Hybrid domain audio watermarking embedding algorithm based on redundant discrete wavelet transform. In: International Conference on Computer Systems, Electronics and Control (ICCSEC); 2017 December 25-27; Dalian, China: IEEE; c2018. p. 1134-1137.

[20] Neyman SN, Pradnyana INP and Sitohang B. A new copyright protection for vector map using FFTbased watermarking. TELKOMNIKA (Telecommunication Comput. Electron. Control). 2014; 12(2):367-378.

[21] Mohsenfar SM, Mosleh M and Barati A. Audio watermarking method using QR decomposition and genetic algorithm. Multimed. Tools Appl. 2015; 74(3): 759-779.

[22] Kavitha KJ and Shan BP. Implementation of DWM for medical images using IWT and QR code as a watermark. In: Conference on Emerging Devices and Smart Systems (ICEDSS); 2017 March 3-4; Mallasamudram, India: IEEE; c2017. p. 252-255.

[23] Katzenbeisser S and Petitolas F. Information hiding techniques for steganography and digital watermaking. Artech House Books; 1999; 28(6). 